\title{
The Study of Interoperability Test for Onboard Control System in Railway Condition
}

\author{
Jonghyen, Baek ${ }^{1, a}$, Yongkyu, Kim ${ }^{2, b}$, Jaeho, Lee ${ }^{3, c}$, Hyenjung, Jo 4,d \\ 1,2,3,4\#360-1, Woram-dong, Uiwang-city, Gyeonggi-do, Korea, Korea Railroad Research Institute \\ ajhbaek@krri.re.kr, 'bgkim1@krri.re.kr, 'prolee@krri.re.kr, ${ }^{\mathrm{d} h j j o @ k r r i . r e . k r(c o r r e s p o n d i n g ~ a u t h o r) ~}$
}

Keywords: TCS(Train Control System), Interoperability, Site Test

\begin{abstract}
For the purpose of improving the future domestic train control systems and securing interoperability, according to the global development trends of train control systems, it is presented that the test results of interoperability between wayside train control systems installed in existed line, and the onboard train control system. Due to the safety-critical characteristics of train systems, the site test in the section where the wayside equipment is installed may lead to a danger against safety. Therefore, by way of constructing a simulation environment of train control systems, the T/R data systems of the equipment for interoperability are confirmed and the interoperability test are obtained by applying these systems to onboard equipment.
\end{abstract}

\section{Introduction}

Although the domestic rail vehicle is made domestically by several firms, in case of train control systems (hereinafter referred to as "TCS"), which is in charge of a rapid and safe passenger transfer, the multinational, multi-product products from foreign suppliers are introduced due to the characteristics of a market and the demand in variability etc. As a result, a different system is used in each route such as high speed rail, general railways, urban transits etc.

For this reason, it experiences operational problems owing to a lack of mutual compatibility and operability specific to devices serving the same functions. Additionally, in case of any accident affecting to safety and convenience of passengers, it is hard to pinpoint the exact cause of an accident and take immediate countermeasure in reality

In order to reduce maintenance costs and to accomplish performance improvement, there is a growing trend for train control systems to be changed from the fixed block systems based on track circuits to the virtual and the moving block systems based on ICT(Information and Communication Technology) radio communication systems[1-7].

Such an train control system with ICT has been actively studied in foreign countries and some parts of the systems have been already commercialized. In order to overcome the situation of train control systems highly dependent on foreign technologies even now, we also must pursue a localization strategy through the technology development for the ICT applied train control system in Korea.

In this paper, we primarily present test contents and results to identify the interoperability between different kinds of TCS in order to meet such a global trend and the needs of the domestic technology development.

Although we should identify the interfaces between onboard and wayside TCS installed in rail vehicles to verify the interoperability. Because of the safety-critical features of railways, if the onboard unit is installed in a real train without interface testing in advance and the trial run is conducted in the section where the different kinds of wayside TCS are installed, it could jeopardize safety. Therefore, we performed the test by configuring test environments[1-9].

\section{Summary of ICT applied TCS}

For the ICT-applied train control system, the computer located in wayside periodically collects positions and speed data from each train and transmits the distance from a preceding train to speed-limited location, to a train. In this way the onboard control units provide the optimal speed control suited for train performance. This system uses radio communication for the onboard-wayside data transmission[1,7]. 
As the wayside railroad crossing controller and the switch point machine are controlled by onboard units, it will be helpful to minimize wayside units in a field site and to increase the efficiency of maintenance. The overall layout for the operation of the ICT based train control systems is shown in figure 1 .

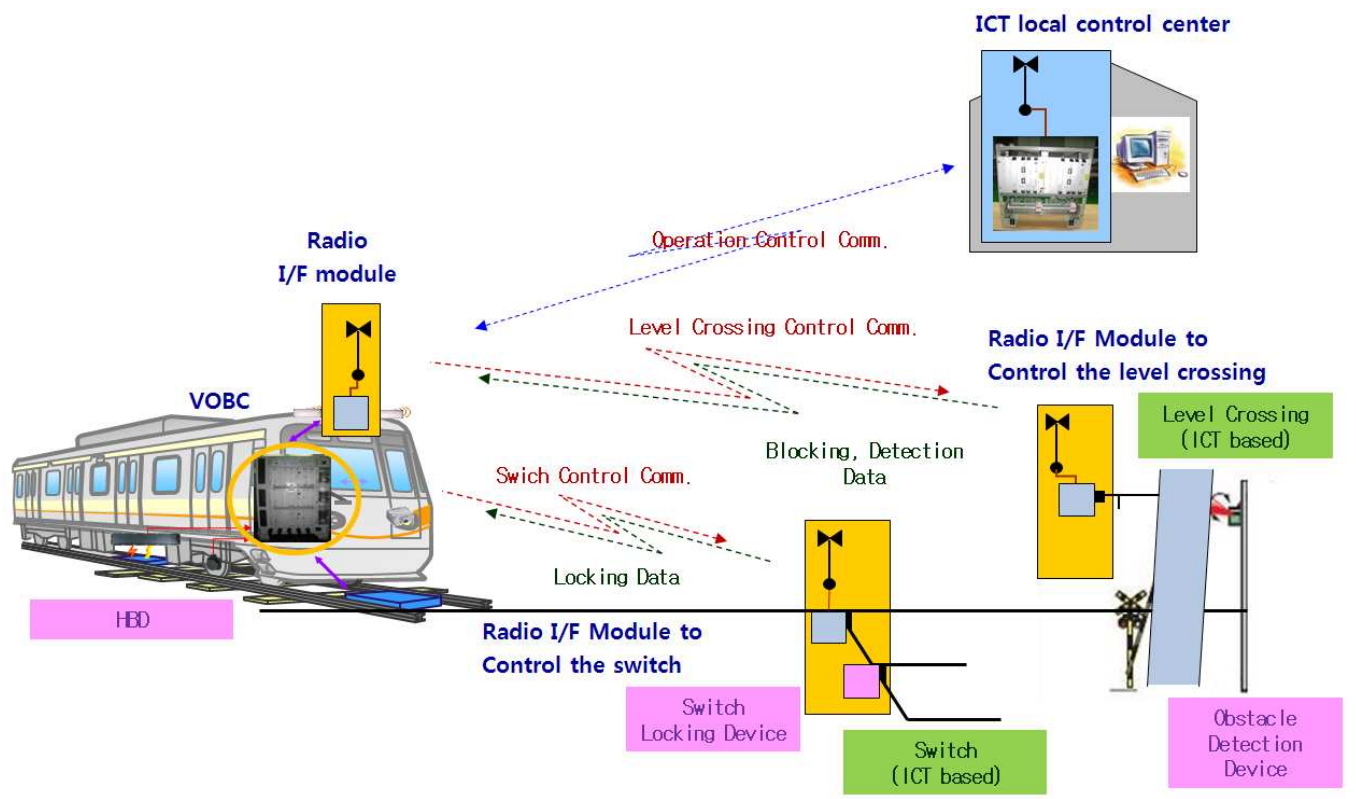

Fig 1. Outline of the ICT based TCS operation

\section{Interoperability Test between different kinds of TCS}

The ICT based TCS has just started its development. For the interoperability test and analysis between different kinds of TCSs presented in this paper, we targeted the different kinds of TCS using radio communication[3, 8-10].

\subsection{Test Environment}

For the interoperability verification of communication based train control systems, we collected the basic onboard-wayside interface data from TCS manufacturers whose products are now installed in an actual onboard and then we established the test environments by leasing Data Communication Units(hereinafter referred to as DCU)

\subsection{Test Configuration}

Figure 2 shows the configuration of the onboard TCS to perform the onboard-wayside interoperability test. Instead of WTP(Wayside Telegram Processor) within DCU, we conducted a test with the wayside TCS with a separate experimental Fake WTP.

Table 1 briefly illustrates about the basic setup values of each specific device used in the interoperability test. Table 2 shows the Routing Table to be set in the Fake WTP. 


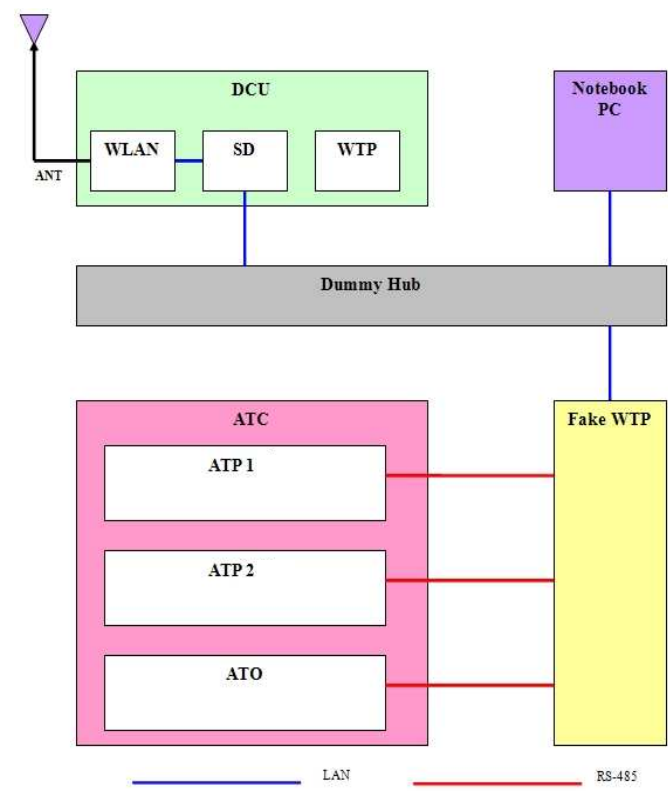

Fig 2. Test Configuration
Table 1 Set-up information specific to devices

\begin{tabular}{|c|c|c|}
\hline \multirow{2}{*}{$\begin{array}{l}\text { Eqpt. } \\
\text { Class. }\end{array}$} & IP & \multirow{2}{*}{ Notes } \\
\hline & MAC & \\
\hline \multirow{2}{*}{ WLAN } & $\begin{array}{c}00: 04: 47: 33: \\
06: 42\end{array}$ & \multirow{2}{*}{ IEEE802.b WLAN } \\
\hline & 172.17.4.2 & \\
\hline \multirow{2}{*}{$\begin{array}{c}\text { SD } \\
\text { (Security } \\
\text { Device) }\end{array}$} & $\begin{array}{c}\text { 00:30:59:01: } \\
\text { 56:A5 }\end{array}$ & \multirow{2}{*}{$\begin{array}{l}\text {-Encryption \& Decryption } \\
\text {-Activated by Router }\end{array}$} \\
\hline & 172.17 .48 .9 & \\
\hline \multirow[b]{2}{*}{$\begin{array}{l}\text { Fake } \\
\text { WTP }\end{array}$} & $\begin{array}{c}00: \mathrm{C} 0: \mathrm{BE}: 01: \\
01: 15\end{array}$ & \multirow{2}{*}{$\begin{array}{l}\text {-WLAN communication with } \\
\text { wayside units } \\
\text {-RS- } 485 \text { communication with } \\
\text { onboard units } \\
\text { - It uses an IP address } \\
\text { (172.17.48.9) of SD as a Default } \\
\text { Gateway }\end{array}$} \\
\hline & 172.17 .48 .10 & \\
\hline \multirow{2}{*}{$\begin{array}{c}\text { Note } \\
\text { book PC }\end{array}$} & $\begin{array}{c}\text { 00:0B:5D:4D: } \\
\text { 98:CE }\end{array}$ & \multirow{2}{*}{$\begin{array}{l}\text { It monitors the communication } \\
\text { traffic between a Fake WTP and } \\
\text { wayside units. }\end{array}$} \\
\hline & 172.17 .48 .100 & \\
\hline
\end{tabular}

Table 2 Setup information of Fake WTP(Routing Table)

\begin{tabular}{|c|c|c|c|c|c|c|c|c|c|c|c|}
\hline \multicolumn{6}{|c|}{ ROUTE NET TABLE } & \multicolumn{6}{|c|}{ ROUTE HOST TABLE } \\
\hline Destination & Gateway & Flag & Recent & Use & Interface & Destination & Gateway & Flag & Recent & Use & Interface \\
\hline 0.0 .0 .0 & 172.17 .48 .9 & $0 \times 103$ & 1 & 0 & cmp0 & 127.0 .0 .1 & 127.0 .0 .1 & $0 \times 5$ & 0 & 0 & 100 \\
\hline 172.17 .48 .0 & 172.17 .48 .10 & $0 \times 101$ & 2 & 0 & $\mathrm{cmp} 0$ & 172.17 .37 .4 & 172.17 .48 .9 & $0 \times 8007$ & 1 & 930 & $\mathrm{cmp} 0$ \\
\hline
\end{tabular}

\subsection{Test Methods}

With use of the Network Packet Monitoring program, we analyzed the transmission packets between onboard and wayside TCSs installed in an actual field site and identified the onboard/wayside IP systems and transmission data. On the basis of this result, we conducted the interoperability test between different kinds of onboard TCSs and the actually installed wayside TCS.

\subsubsection{Communication with ATS(Automatic Train Supervision)}

The WTP-ATS communication is based on the UDP communication and the UDP port is 25910. It is normally operated at the IP address of [172.17.37.4]. Table 3 briefly shows the types of communication tests between WTP and ATS

Table 3 Summary of ATS Comms Test Items

\begin{tabular}{|c|c|c|c|}
\hline Items & CRC & $\begin{array}{c}\text { Initiator } \\
\text { Sequence Number }\end{array}$ & Notes \\
\hline A & Fixed CRC & 0 & $\begin{array}{l}\text { Communication packets extracted from the real communication data } \\
\text { between previously captured WTP and ATS }\end{array}$ \\
\hline B & Fixed CRC & Values except 0 & $\begin{array}{l}\text { Communication packets extracted from the real communication data } \\
\text { between previously captured WTP and ATS }\end{array}$ \\
\hline C & $\begin{array}{c}\text { Calculated } \\
\text { CRC }\end{array}$ & 0 & Communication packets produced by dynamically computing CRC-32 \\
\hline
\end{tabular}

\subsubsection{Communication with WCU}

The WTP-WCU communication is based on the UDP communication and the UDP port is 25910. It is normally operated at the IP address of [172.17.40.34]. Table 4 briefly shows the types of communication tests between WTP and WCU. 
Table 4 Summary of WCU Comms Test Items

\begin{tabular}{|c|c|c|c|}
\hline Items & CRC & $\begin{array}{c}\text { Sequence No. } \\
\text { of Initiator }\end{array}$ & Notes \\
\hline A & Fixed CRC & 0 & $\begin{array}{l}\text { Communication packets extracted from the real communication data } \\
\text { between previously captured WTP and WCU }\end{array}$ \\
\hline B & Fixed CRC & Values except 0 & $\begin{array}{l}\text { Communication packets extracted from the real communication data } \\
\text { between previously captured WTP and WCU }\end{array}$ \\
\hline C & $\begin{array}{c}\text { Calculated } \\
\text { CRC }\end{array}$ & 0 & $\begin{array}{l}\text { Communication packets produced by } \\
\text { dynamically computing CRC-32 }\end{array}$ \\
\hline
\end{tabular}

\section{Test Results \& Analysis of Communication Interfaces}

\subsection{Communication Test Results with ATS}

The following is the summary of the communication test results of the Fake WTP and ATS

Table 5 Summary of Comms Test Results with ATS

\begin{tabular}{|c|l|l|}
\hline Items & \multicolumn{1}{|c|}{ Test Results } & \multicolumn{1}{c|}{ Notes } \\
\hline A & $\begin{array}{l}\text { Normal } \\
\text { communications }\end{array}$ & Two-way Communications operation (Communications Cycle is 500ms) \\
\hline B & $\begin{array}{l}\text { Communications } \\
\text { Failure }\end{array}$ & ATS not responding \\
\hline C & $\begin{array}{l}\text { Communications } \\
\text { Failure }\end{array}$ & ATS not responding \\
\hline
\end{tabular}

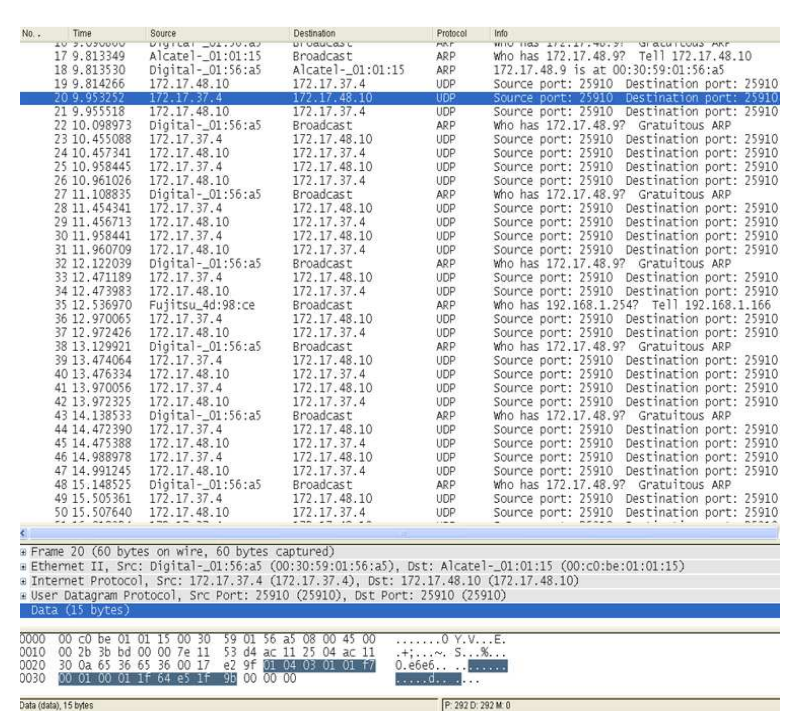

Fig 3. Comms Test Results with ATS (Test A, Ethereal) $\rightarrow$ RecvFmats: Rx(devname=usock1), len=15

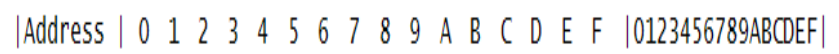

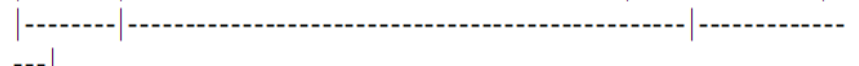

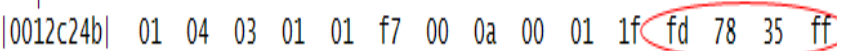
$\times 5.1$

SendToAts: Tx(deuname=usock1), len=28

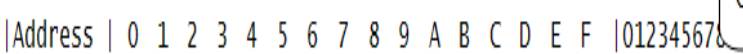
|---.--

$---\mid$

$\begin{array}{lllllllllllllllll}\text { |0012be41| } & 03 & 01 & 01 & 04 & 00 & 00 & 01 & f 7 & 00 & 0 e & c 0 & 00 & 6 c & 00 & 00 & \text { f8 }\end{array}$ $\mid$...............

|0012be51| 000010000000000022 ce ea e0

Fig 4. Comms Test Results with ATS (Test A, Fake FTP Console-log)

As a result of the Network Packet Monitoring via the Ethereal Network Protocol Analyzer, Figure 3 shows that the T/R of Poll Messages is in progress by $500 \mathrm{~ms}$ between a Fake WTP and ATS. As a result of identifying the communication status between WTP and ATS through Fake WTP console ports, we verified that the two-way communication between a Fake WTP and ATS is in progress(see Figure 4).

We verified the Network Packet Monitoring for test B and C along with a WTP-ATS communication status through console ports of a Fake WTP, we identified that there was no response from ATS against the packet initiating communication out of Fake WTP. 


\subsection{Communication Test Results with WCU}

The communication test result between a Fake WTP and WCU is outlined in the following table. The result of a Test $\mathrm{A}$ shows that Test $\mathrm{B}$ and $\mathrm{C}$ were not conducted because the communication between a Fake WTP and WCU has not initiated.

Table 6 Summary of the Comms Test Results with WCU

\begin{tabular}{|c|c|l|}
\hline Items & \multicolumn{1}{|c|}{ Test Results } & \multicolumn{1}{c|}{ Notes } \\
\hline A & $\begin{array}{l}\text { Communications } \\
\text { Failure }\end{array}$ & WCU not responding \\
\hline B & - & No Progress \\
\hline C & - & No Progress \\
\hline
\end{tabular}

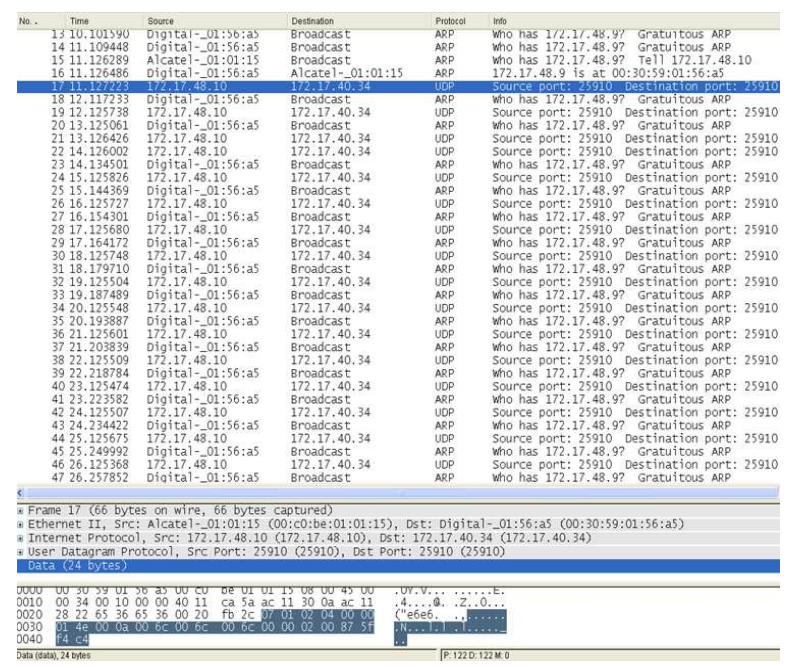

Fig 5. Comms Test Results with WCU (Test A, Ethereal)
SendTowatp: Tx (devname=usock0), len=24

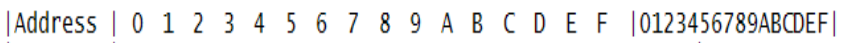

$1--$

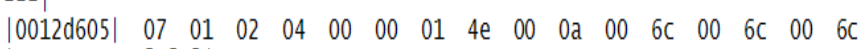
|...........1.1.1|

|0012d615| $00000200875 f$ f4 C4

RecvFmwatp: devname=usock0, HttRecv() timeout !!!

SendTowatp: Tx(devname=usock0), len=24

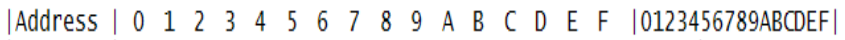

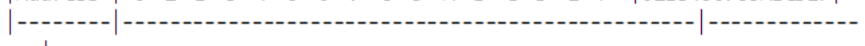

$---\mid$

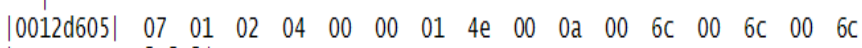
|...........1.1.1|

|0012d615| $00000200875 f$ f4 C4

RecvFmwatp: devname=usock0, HttRecv() timeout !!!

Fig 6. Comms Test Results with WCU

(Test A, Fake FTP Console-log)

As a result of a Network Packet Monitoring, it can be found that there was no response from WCU against the packet initiating communication out of the Fake WTP(see Figure 5).

But, due to the fact that ICMP packets from SD operated by a Router, for example Destination unreachable(Host unreachable)" or "Destination unreachable(Port unreachable)" etc, were not received, it can be regarded that the packets transmitted from a Fake WTP are normally forwarded to the application program operated in WCU units at the least way.

As a result of identifying the WTP-WCU communication status through Fake WTP console ports, it can be found that there is no response from WCI against the packet initiating communication out of Fake WTP(see Figure 6).

\subsection{Analysis on the Test Results of Communication Interfaces}

The result of the communication test with ATS using a Fake WTP shows that the normal communication is available with use of CRC-32 figures, previously verified. However, in case that the communication is attempted after yielding CRC-32 values by applying the well-known CRC-32 calculation method, the ATS does not response. Next, the WCU communication test result with use of a Fake WTP shows that there is no response from WCU in spite of the use of the previously verified CRC-32. It can be considered that the information of the blocks (using TAG ID) that are currently occupied by a train should be exchanged first of all.

Consequently, we verified that, if a separate Fake WTP is used, the basic WLAN communication to the wayside ATS is available via SD that is an encipherment unit within DCU. In addition, we must, first, acquire the verified calculation method of CRC-32, which is commonly used in the wayside ATS and WCU in order to establish the normal communication between WTP/ATS and WTP/WCU. 


\section{Conclusion}

The interoperability tests between WCU-ATS units and a different kind of onboard train control system are performed with the help of the basic interfaces for the interoperability test from the provider manufacturing wayside train control systems. The test result shows that it is impossible to realize the interoperability in case that the wayside and onboard manufacturers are different without sharing the whole information like wireless communication cryptosystem and key values for communication, transmission speed, data formats and CRC formula, IP Network system etc.

In other words, as the configuration logic of the information and communication data is regarded as the unique source technology of manufacturers in terms of safety and reliability, it is expected that the interoperability of different kinds of TCS is very difficult. For the interoperability of trains, it is very meaningful that the reception of the frequency transmitted by the same transmission system within a simply given frequency range allows the mutual communication to be established.

Accordingly, the information data for the train operation should be safely received, then it is required to utilize the data in onboard units and such a series of process should be safely informed to wayside systems. And the interoperability can be satisfied only when the coherence and consistency of information data between different kinds of facilities are accurately implemented. For this reasons, the localization of the integrated onboard and wayside TCS is necessary. The safety and the reliability should be secured by a continuous trial run and testing as well. Consequently, the dedicated test lines for the trial operation of the developed systems must be acquired primarily.

\section{References}

[1] Jonghyun Baek, Kangmi Lee, Youngkyu Kim, "Testing and analysis of Communication interfaces for the verification of TCS interoperability", Journal of the Korean Academic Industrial Society, Vol 11, Iss 11, pp.4496-4502, 2010.

[2] Jonggi Kim, Jonghyun Baek et al, "R/D project for the standardization of Metro signal systems", KRRI R/D Report 2005, MOCT

[3] Young-tae Kim(2003), "Signal Control System", Techno Media, pp.446-469

[4] KORAIL, AKRSE(2004), "Handbook of Railway Signaling Terms"

[5] Jonggi Kim, Jonghyun Baek et al(2006), "R/D project for the standardization of Metro signal systems", the R/D Final Report for the standardization of Metro signal systems", MOCT

[6] Yongki Kim, Yongkyu Kim et al(2004), "Train Control System Using Wireless Communication", Journal of the Korean Society for Railway, Vol 7, Iss 2, pp.22-28.

[7] MOCT(2003), "Rail Transportation Policy using MBS"

[8] Jonghyun Baek, Yongkyu Kim et al, "Analysis of EMU Installation and Yard Test for Communication Based Train Control On-board Equipment", Journal of the Korean Academic Industrial Society, Vol 10, Iss 5, pp.935-941, 2009.

[9] Samsung SDS, KORAIL(2008), "the Final Report of the Trial Project for the Intelligent Train Control System (MBS) at Korail Bundang Line"

[10] Peterson, W. W, Brown, D. T. "Cyclic Codes for Error Detection." Proceedings of the IRE, pp.228-235, 1961. 